\title{
Adaptación y Análisis Psicométrico de la Versión Española del Índice Internacional de Función Eréctil (IIEF) en Población Chilena.
}

\section{Adaptation and Psychometric Analysis of the Spanish Version of the International Index of Erectile Function in Chilean Population}

\author{
Rodolfo Hernández \\ Tatiana Thieme \\ Universidad de Concepción, Chile. \\ Fernando Araos \\ Universidad del Desarrollo, Chile. \\ Director Centro Chileno de Sexualidad, Chile. \\ Recepcionado (8 de junio 2015) Aceptado (29 de julio 2016)
}

\begin{abstract}
Resumen
Contar con instrumentos validados en Chile para Disfunción Eréctil (DE), motivó el validar la versión española del Índice Internacional de Función Eréctil (IIEF), en población chilena. Se realizó un estudio cuantitativo no experimental transversal, de muestra no probabilística por conveniencia, con 95 varones adultos de tres centros de salud (Concepción, Talcahuano y Santiago), durante Noviembre 2012 y Junio 2013. Expertos ciegos realizaron entrevista clínica utilizando criterios DSM-V y causas médico-quirúrgicas de DE. Luego de aplicación piloto y adaptación del IIEF, se logró la versión IIEF-Ch aplicándola a toda la muestra. Se analizaron los resultados con SPSS 15.0, obteniéndose alta correlación entre variables $\left(\mathrm{X}^{2}: 956.533\right)$ y alta adecuación muestral $(0,893)$; dos factores que explican el $81.59 \%$ de la varianza del instrumento, con alta confiabilidad (.971) y adecuada validez de criterio, alta sensibilidad (84.5\%) y especificidad aceptable (91.9\%). La IIEF-Ch es un instrumento válido y confiable para estudios sobre DE en Chile.

Palabras claves: Entrevista diagnóstica, Índice Internacional de Función Eréctil, Disfunción Eréctil.
\end{abstract}

\begin{abstract}
Having instruments validated in Chile for Erectile Dysfunction (ED), motivated the validation of the Spanish version of the International Index of Erectile Function (IIEF), in Chilean population. A non-experimental quantitative study of non-probabilistic sample was carried out for convenience, with 95 adult males from three health centers (Concepción, Talcahuano and Santiago), during November 2012 and June 2013. Blind experts conducted a clinical interview using DSM-V criteria and medical-surgical causes of ED. After the pilot application and adaptation of the IIEF, version IIEF-Ch was achieved by applying it to the entire sample. The results were analyzed with SPSS 15.0, obtaining high correlation between variables $\left(\mathrm{X}^{2}: 956,533\right)$ and high sample adequacy $(0,893)$; two factors that explain $81.59 \%$ of the variance of the instrument, with high reliability (.971) and adequate criterion validity, high sensitivity (84.5\%) and acceptable specificity (91.9\%). The IIEF-Ch is a valid and reliable instrument for studies on ED in Chile.

Key words: Diagnostic interview, International Index of Erectile Function, Erectile Dysfunction.
\end{abstract}

Correspondencia: Rodolfo Hernández S., Departamento de Psiquiatría y Salud Mental, Facultad de Medicina, Universidad de Concepción. Teléfono: +56-41 2204299. E-mail: rodhersil@gmail.com 


\section{Introducción}

La DE es una disfunción sexual mencionada reiteradamente en la literatura médica así como en la psicoterapéutica. Los estudios internacionales disponibles demuestran que la frecuencia de la disfunción eréctil es relevante, sin embargo, en la literatura científica chilena, no se registran estudios de prevalencia realizados con escalas validadas, por lo que no se conoce la real frecuencia de la patología en Chile.

Según Laumann, Paik, y Rosen (1999), en países de Europa y Asia se mencionan cifras altas de las disfunciones sexuales en general, que van de entre $10 \%$ a $52 \%$ en varones y de $25 \%$ a $63 \%$ en mujeres. De acuerdo a McVary (2011) la disfunción sexual masculina afecta a una proporción de 10 a $25 \%$ de los varones de edad madura y avanzada. Según el estudio de Envejecimiento Masculino de Massachusetts realizado entre los años 1987 y 1989, en hombres de entre 40 a 70 años, la DE surge como una entidad nosológica relevante que afecta a más de 100 millones de personas en el mundo, aproximadamente el $10 \%$ de la población en cualquier edad la padece siendo mayor a medida que la edad aumenta: $40 \%$ a los 40 años, $50 \%$ a los 50 años y $60 \%$ a los 60 años (Feldman en Kloner, 2005).

El propósito de este estudio es validar la versión española del IIEF en población chilena, con el fin de contar con un instrumento válido para estudiar la prevalencia en Chile de DE, complementar métodos diagnósticos y apoyar la terapéutica médica y psicológica.

\section{Método}

Se ejecutó un estudio de carácter metodológico, empleando un diseño de tipo no experimental-transversal (Hernández, Fernández y Baptista, 2006).

\section{Participantes}

Los participantes fueron varones adultos hospitalizados en el Servicio de Urología del Hospital Guillermo Grant Benavente de Concepción y en el Servicio de Urología del Hospital las Higueras de Talcahuano, y pacientes consultantes ambulatorios al Centro Chileno de Sexualidad, con y sin DE.

Se realizó un muestreo aleatorio de participantes voluntarios que consultaron a los centros de salud mencionados. Se seleccionaron pacientes por conveniencia, a partir de la evaluación clínica realizada por especialistas Urólogos, quienes realizaron el diagnóstico de las posibles causas médico-quirúrgicas de la DE, y por Psiquiatra y Psicólogo, los que aplicaron una evaluación clínica utilizando los criterios diagnósticos del Manual Diagnóstico y Estadístico de los Trastornos Mentales (DSM-V) para diagnosticar DE.

La muestra total estuvo constituida por 95 varones, con edades entre 25 y 88 años, con una media de 54 años (ds 15.5 años). Configurándose un grupo de casos (52 participantes diagnosticados con DE) y un grupo control (43 participantes sin diagnóstico de $\mathrm{DE}$, seleccionados en base a las características sociodemográficas del grupo de casos). Se encuestó a todos los sujetos.

\section{Instrumento}

Se empleó la escala IIEF, versión española, que según Corona, Jannini y Maggi (2006), ha sido ampliamente utilizada en estudios de función eréctil masculina. La escala original fue desarrollada por el equipo formado por Rosen, Riley, Wagner, Osterloh, Kirkpatrick y Mishra (1997), con la intención de crear un instrumento breve, psicométricamente válido y de auto aplicación, minimizando el sesgo de deseabilidad social de la heteroaplicación. Consta de 15 ítems que evalúan la presencia de problemas en las distintas fases de la respuesta sexual humana (deseo, erección, eyaculación y orgasmo), junto con la satisfacción en la relación sexual y satisfacción global.

Para contestar a cada ítem, el paciente ha de utilizar una escala tipo Likert, unas veces de frecuencia, otras de intensidad, y otras de satisfacción (García-Portilla, Bascarán, Sáiz, Parellada, Bousoño y Bobes , 2002). El marco de referencia temporal son las 4 últimas semanas. La escala es de auto reporte, con 15 ítems con un formato de respuesta tipo Likert de 5 puntos cada uno.

Inicialmente, se realizó un estudio piloto, posteriormente, se adaptó el lenguaje utilizado en la escala IIEF española, logrando una adecuación a la idiosincrasia de la población chilena, resultando el IIEF-Ch

\section{Procedimiento}

Se trabajó con juicio de experto para comparar los diagnósticos clínicos con el resultado en el IIEF-Ch de cada encuestado. El juicio de experto se logró mediante la realización de entrevistas clínicas por psicólogo y psiquiatra, utilizando los criterios diagnósticos del DSM-V para DE, además los pacientes fueron evaluados por urólogos, para determinar causas médico-quirúrgicas de DE. 
Para llevar a cabo el estudio, se tomó contacto con los jefes de servicio del Servicio de Urología del Hospital Guillermo Grant Benavente de Concepción, del Servicio de Urología del Hospital las Higueras de Talcahuano, y con miembros del Centro Chileno de Sexualidad, para obtener aprobación por el Comité de Ética de cada institución y colaboración en el estudio.

Cada paciente firmó un consentimiento informado antes de participar en la investigación, seguido inmediatamente por la realización de la entrevista clínica a cada participante del estudio y la posterior aplicación del IIEF-Ch.

\section{Análisis de datos}

Los datos fueron analizados mediante el paquete estadístico SPSS 15.0. Para estudiar la estructura de la escala IIEF-Ch, se empleó el método de los componentes principales (ACP) para la extracción de los factores. Para obtener la validez de los resultados, se utilizó el Índice de adecuación muestral Kaiser-Meyer-Olkin (KMO) y el test de esfericidad de Bartlett.

\section{Resultados}

Del total de sujetos estudiados, un $54.7 \%$ presentaba DE y el $55.8 \%$ de este grupo presentaba patología médica, siendo la más frecuente la patología prostática (30.6\%), lo que se desglosa en adenoma prostático (25.3\%), cáncer prostático $(5.3 \%)$, hipertensión arterial (14.7\%), y diabetes mellitus (10.5).

La validez de los resultados de un ACP viene condicionada a obtener valores significativos (próximos a una unidad) del índice de adecuación muestral Kaiser-Meyer-Olkin (KMO) $\mathrm{y}$ el test de esfericidad de Bartlett $(\mathrm{p}<.05)$ que obtiene de la matriz de correlaciones. En nuestro caso, ambos valores son favorables $\left(\mathrm{KMO}=0.893\right.$; Bartlett, $\mathrm{X}^{2}$ : 956.533), haciendo factible la aplicación de la ACP a la IIEF-Ch, obteniéndose una matriz de dos componentes, los que se presentan en la tabla 1.

El factor número 1, al cual se le asignó el nombre de "Capacidad eréctil-orgásmica satisfactoria"(CEOS) agrupa los ítems relacionados con la función eréctil, función orgásmica, satisfacción de la relación y satisfacción global (64.62\%) y

Tabla 1. Matriz de Factores del IIEF-Ch de la Población Total Estudiada

\begin{tabular}{llll}
\hline & & \multicolumn{2}{l}{ Componente } \\
& & $\mathbf{1}$ & \multicolumn{2}{l}{$\mathbf{2}$} \\
\hline 1. & ¿Con qué frecuencia logro una erección durante la actividad sexual? & 0.824 & 0.341 \\
2. & ¿Con qué frecuencia fue suficiente la rigidez de su erección para la penetración? & 0.892 & 0.233 \\
3. & ¿Al intentar la penetración sexual, con qué frecuencia lo logró? & 0.953 & 0.16 \\
4. & ¿Con qué frecuencia logró mantener la erección después de la penetración? & 0.94 & 0.207 \\
5. ¿Cuál fue el grado de dificultad para mantener la erección hasta completar la relación sexual? & 0.945 & 0.179 \\
6. & ¿Cuántas veces intentó la penetración sexual? & 0.755 & 0.241 \\
7. & ¿Con qué frecuencia resultó satisfactorio para usted? & 0.917 & 0.27 \\
8. ¿Cuánto ha disfrutado de la penetración sexual? & 0.922 & 0.234 \\
9. & ¿Durante la actividad sexual, con qué frecuencia eyaculó? & 0.856 & 0.285 \\
10. ¿Durante la actividad sexual, con qué frecuencia tuvo una sensación de orgasmo? & 0.843 & 0.311 \\
11. ¿Cómo calificaría su nivel de deseo de tener una experiencia sexual? & 0.149 & 0.922 \\
12. ¿Con qué frecuencia ha sentido el deseo de tener una experiencia sexual? & 0.185 & 0.891 \\
13. ¿Cuál ha sido el grado de satisfacción con su vida sexual en general? & 0.534 & 0.448 \\
14. ¿Cuál ha sido el grado de satisfacción experimentado en la penetración sexual? & 0.917 & 0.256 \\
15. ¿Cómo calificaría la confianza que tiene en poder lograr y mantener una erección? & 0.584 & 0.554 \\
\hline
\end{tabular}

Nota. ítems 1 al 10 más 13 al 15: Factor 1; ítems 11 y 12: Factor 2. 
el factor número 2, al cual se le asignó el nombre de "Deseo Sexual" (DSex), agrupa los ítems relacionados con el deseo sexual (16.96\%). Ambos factores logran explicar el $81.59 \%$ de la varianza del instrumento.

El alfa de Cronbach obtenida para la muestra total es de 0.971, considerada como excelente medida de fiabilidad del IIEF-Ch. Para el factor CEOS, es de 0.978 y el factor DSex, es de 0.861 , éste último coincide con lo descrito por los autores originales.

Los resultados de validez de criterio para el total de la muestra, considerando el juicio clínico como patrón de referencia (gold standard), se presentan en la tabla 2.

Tabla 2. Sensibilidad, Especificidad y Puntaje de Corte del IIEF-Ch

\begin{tabular}{lllll}
\hline & \multicolumn{4}{c}{ Juicio Experto } \\
& & Positivo & Negativo & Total \\
\hline \multirow{2}{*}{ Bajo 41 } & Numero de sujetos & 49 & 3 & 52 \\
ptos. & \% del N & $94.2 \%$ & $5.8 \%$ & $100.0 \%$ \\
& \% de Juicio Experto & $84.5 \% *$ & $8.1 \%$ & $54.7 \%$ \\
\multirow{2}{*}{41 y } & Numero de sujetos & 9 & 34 & 43 \\
mas & \% de N & $20.9 \%$ & $79.1 \%$ & $100.0 \%$ \\
\multirow{2}{*}{ Total } & \% de Juicio Experto & $15.5 \%$ & $91.9 \% * *$ & $45.3 \%$ \\
& Numero de sujetos & 58 & 37 & 95 \\
& \% de N & $61.1 \%$ & $38.9 \%$ & $100.0 \%$ \\
& \% de Juicio Experto & $100.0 \%$ & $100.0 \%$ & $100.0 \%$ \\
\hline
\end{tabular}

Nota. * Sensibilidad, ** Especificidad

El instrumento reveló una alta sensibilidad para diagnosticar disfunción eréctil, el $83.3 \%$ de los sujetos identificados por la prueba como pacientes con disfunción eréctil presentaron el mismo diagnóstico frente al juicio de experto. La especificidad es aceptable (91.2\%), con lo que el instrumento tiene la capacidad suficiente para detectar ausencia de disfunción eréctil en sujetos sanos. Con estos datos se determinó que el puntaje de corte del IIEF-Ch es de 41 puntos, así, 40 puntos o menos es indicativo de DE.

Se determinaron rangos de intensidad de DE para el IIEF-Ch. Un puntaje bruto de 0 a 11 corresponde a una DE severa, de 12 a 23 a DE moderada, de 24 a 40 DE leve y de 41 a 75 corresponde a ausencia de DE.

El factor CEOS, reveló una alta sensibilidad para detectar DE, así, el $89.7 \%$ de los sujetos identificados con CEOS bajo lo normal, presentaron el diagnóstico de DE según juicio de experto, junto a ello la especificidad demostró ser aceptable (89.2\%).

El factor DSex, reveló una alta sensibilidad para detectar DE, de tal modo que el $70.7 \%$ de los sujetos identificados por la prueba como pacientes con DSex disminuido, presentaron DE frente al juicio de experto, además, la especificidad resultó ser aceptable para este factor $(70.3 \%)$.

La mayoría de los encuestados con DE presenta un DSex muy bajo, moderadamente bajo o levemente bajo. Por otro lado, la mayoría de los encuestados sin DE, presentan un DSex adecuado (60.5\%), con una significación estadística de $\mathrm{x}^{2} 0.001$. Existe una relación estadísticamente significativa entre DSex y DE, así, con DSex adecuado, hay menor probabilidad de presentar DE, tal como lo muestra la tabla 3.

$\mathrm{Al}$ analizar el DSex según causa orgánica o psicógena de la DE, encontramos que en el $18.6 \%$ de los encuestados con DSex adecuado presentan causa orgánica, mientras que el $81.4 \%$ presenta causa psicógena $\left(\mathrm{x}^{2} 0.020\right)$. Es decir, existe una relación estadísticamente significativa entre DSex y la causa de la DE. Así, con DSex adecuado, existe mayor probabilidad de que la DE sea de causa psicógena.

Al analizar el DSex en relación a la edad, encontramos que el DSex muy bajo se encuentra en mayor proporción en

Tabla 3. Deseo Sexual y Disfunción Eréctil

\begin{tabular}{llllllll}
\hline & & \multicolumn{4}{l}{ Disfunción eréctil } & \multicolumn{3}{l}{} \\
& & \multicolumn{2}{l}{ Positivo } & \multicolumn{2}{l}{ Negativo } & \multicolumn{2}{l}{ Total } \\
& & $\mathrm{N}$ & $\%$ & $\mathrm{~N}$ & $\%$ & $\mathrm{~N}$ & $\%$ \\
\hline \multirow{3}{*}{ Deseo sexual } & 12 & $85.7 \%$ & 2 & $14.3 \%$ & 14 & $100.0 \%$ \\
& Muy bajo & 14 & $82.4 \%$ & 3 & $17.6 \%$ & 17 & $100.0 \%$ \\
& Moderadamente bajo & 15 & $71.4 \%$ & 6 & $28.6 \%$ & 21 & $100.0 \%$ \\
& Levemente bajo & 17 & $39.5 \%$ & 26 & $60.5 \%$ & 43 & $100.0 \%$ \\
& Deseo sexual adecuado & 58 & $61.1 \%$ & 37 & $38.9 \%$ & 95 & $100.0 \%$ \\
\hline
\end{tabular}


Tabla 4. Deseo Sexual y Causas de Impotencia

\begin{tabular}{llllllll}
\hline & & \multicolumn{3}{l}{ Disfunción eréctil } & & \\
& & Orgánico & \multicolumn{2}{l}{ Psicógeno } & \multicolumn{2}{l}{ Total } \\
& & $\mathrm{N}$ & $\%$ & $\mathrm{~N}$ & $\%$ & $\mathrm{~N}$ & $\%$ \\
\hline \multirow{3}{*}{ Deseo } & Muy bajo & 7 & $50.0 \%$ & 7 & $50.0 \%$ & 14 & $100.0 \%$ \\
sexual & Moderadamente bajo & 9 & $52.9 \%$ & 8 & $47.1 \%$ & 17 & $100.0 \%$ \\
& Levemente bajo & 5 & $23.8 \%$ & 16 & $76.2 \%$ & 21 & $100.0 \%$ \\
& Deseo sexual adecuado & 8 & $18.6 \%$ & 35 & $81.4 \%$ & 43 & $100.0 \%$ \\
Total & & 29 & $30.5 \%$ & 66 & $69.5 \%$ & 95 & $100.0 \%$ \\
\hline
\end{tabular}

Tabla 5. Deseo Sexual y Edad del Entrevistado

\begin{tabular}{|c|c|c|c|c|c|c|c|c|c|c|c|}
\hline & & \multicolumn{10}{|c|}{ Edad del Entrevistado (años) } \\
\hline & & \multicolumn{2}{|c|}{25 a 44} & \multicolumn{2}{|c|}{45 a 58} & \multicolumn{2}{|c|}{59 a 73} & \multicolumn{2}{|c|}{74 y más } & \multicolumn{2}{|c|}{ Total } \\
\hline & & $\mathrm{N}$ & $\%$ & $\mathrm{~N}$ & $\%$ & $\mathrm{~N}$ & $\%$ & $\mathrm{~N}$ & $\%$ & $\mathrm{~N}$ & $\%$ \\
\hline \multirow{4}{*}{ Deseo Sexual } & Muy bajo & 1 & $7.1 \%$ & 4 & $28.6 \%$ & 4 & $28.6 \%$ & 5 & $35.7 \%$ & 14 & $100.0 \%$ \\
\hline & Moderadamente bajo & 3 & $17.6 \%$ & 3 & $17.6 \%$ & 10 & $58.8 \%$ & 1 & $5.9 \%$ & 17 & $100.0 \%$ \\
\hline & Levemente bajo & 3 & $14.3 \%$ & 8 & $38.1 \%$ & 8 & $38.1 \%$ & 2 & $9.5 \%$ & 21 & $100.0 \%$ \\
\hline & Deseo sexual adecuado & 18 & $41.9 \%$ & 16 & $37.2 \%$ & 8 & $18.6 \%$ & 1 & $2.3 \%$ & 43 & $100.0 \%$ \\
\hline Total & & 25 & $26.3 \%$ & 31 & $32.6 \%$ & 30 & $31.6 \%$ & 9 & $9.5 \%$ & 95 & $100.0 \%$ \\
\hline
\end{tabular}

el grupo de mayor edad (74 y más años), con una $\mathrm{x}^{2}$ del 0.01 . Es decir, existe una relación estadísticamente significativa entre DSex y edad, siendo este menor a mayor edad.

Por lo tanto, se puede afirmar que el DSex adecuado es más frecuente en ausencia de DE, en DE de causa psicógena y en sujetos más jóvenes.

\section{Discusión}

Los resultados muestran que el IIEF-Ch es un instrumento internamente consistente, demostrando una excelente coherencia en torno al concepto de Disfunción Eréctil. Respecto del análisis factorial, se lograron dos factores explicativos de la Disfunción Eréctil, a diferencia del trabajo original (Rosen et al., 1997) que identifica cinco factores. Así, en la población estudiada, los reactivos que corresponden a las dimensiones Capacidad Eréctil, Capacidad Orgásmica, y Satisfacción, constituyen un solo factor, mientras que la dimensión Deseo Sexual aparece como el otro factor.

Respecto de la sensibilidad del instrumento para diag- nosticar DE, los resultados catalogan al instrumento como una excelente herramienta para su detección, de tal modo que la gran mayoría de los sujetos identificados por la escala como pacientes con disfunción eréctil, presentaron el mismo diagnóstico al compararlo con los diagnósticos encontrados mediante evaluación clínica realizada por urólogo, psicólogo y psiquiatra, con uso de criterios DSM-IV (juicio de experto). La especificidad encontrada se considera en rango de aceptable, lo que indica que el instrumento tiene la capacidad suficiente para detectar ausencia de disfunción eréctil en sujetos sanos. Con estos datos se determinó el puntaje de corte para el IIEF-Ch con el que se diagnostica DE. Un resultado interesante, reveló que el factor DSex es escasamente relevante en la escala, y que el factor CEOS por sí solo constituye el factor esencial para el diagnóstico de DE (desde un punto de vista exclusivamente estadístico).

En este estudio, el DSex es significativamente menor en pacientes con DE, en pacientes con DE de causa orgánica, y en pacientes de mayor edad, y por el contrario, es significativamente mayor en encuestados $\sin \mathrm{DE}$, en pacientes con $\mathrm{DE}$ 
psicógena, y en encuestados de menor edad. Los resultados encontrados apoyan la relevancia de estudiar elementos psicológicos como el deseo, mediante las nuevas escalas que se pudiesen diseñar para el estudio de la DE, y a su vez hablan de que no sería adecuada la utilización de escalas que no incluyan la evaluación del deseo (escalas abreviadas de DE). De hecho en el presente análisis estadístico, se demuestra que el DSex se mantiene más alto en DE psicógena, en pacientes de menor edad, y en sujetos sin DE. Resulta especialmente interesante desde el punto de vista psicodinámico que el deseo se mantenga mayor en DE psicógena y esto debiese ser considerado en la terapéutica psicológica, y a su vez sería relevante el hecho de que el deseo sea menor en pacientes con DE orgánica, ya que podría estar incluso orientando hacia la necesidad del estudio médico orgánico en estos pacientes especialmente.

De hecho, en este estudio encontramos diferencias estadísticamente significativas al relacionar Dsex con otros parámetros de los encuestados. Estos últimos datos pudiesen ser de utilidad para complementar el diagnóstico con escalas, para especificar el diagnóstico clínico de DE, y eventualmente para orientar la psicoterapia de la $\mathrm{DE}$, y por lo demás podría plantearse que el deseo es un fenómeno psicodinámico y psicopatológico que pudiese considerarse en los manuales diagnósticos como el DSM en cuanto a DE (no se considera el deseo en los criterios diagnósticos DSM-IV ni DSM-V), considerando que el análisis del deseo sexual pudiese tener una posible trascendencia en la terapéutica tanto médica como psicológica.

Según lo encontrado en este estudio, se plantea la posibilidad de entender a la función eréctil en la población estudiada como un todo indivisible entre erección, orgasmo y satisfacción, sin embargo, el deseo se conceptualiza en una dimensión distinta. Esto es interesante, y viene a plantear nuevos desafíos en relación al tema, que probablemente puede abrir nuevas líneas de investigación, ya no sólo la de detectar la DE, sino la de comprender los procesos que subyacen a la función eréctil y sus dificultades, en especial el tema de la comprensión subjetiva del deseo, y de diferenciar la erección propiamente tal, del orgasmo y satisfacción. De hecho, los resultados del presente estudio demuestran que al no considerar los reactivos que pretenden evaluar deseo sexual, aumenta la sensibilidad del IIEF-Ch para detectar sujetos con DE. Esto último, podría explicarse en el sentido de que el deseo, dentro de las dimensiones estudiadas, es la que tiene menos correlato en la conducta sexual masculina físicamente observable, en otras palabras parece ser que los sujetos pueden responder mejor ante un instrumento con reactivos sobre la erección propiamente tal, también sobre la eyaculación, orgasmo y satisfacción (y estos tres últimos, posiblemente algunos de los sujetos entrevistados pudiesen incluso considerarlos como un sinónimo, lo que dificulta la mejor comprensión de la DE).

En conclusión, y en base a los resultados del presente estudio realizado, se puede afirmar que el IIEF-Ch es una escala válida para utilizar en estudios de $\mathrm{DE}$ en la población chilena.

\section{Referencias}

American Psychiatric Association. (2013). Diagnostic and statistical manual of mental disorders ( $5^{\text {th }}$ ed.). Arlington, VA: American Psychiatric Publishing.

Corona, G., Jannini, E A., y Maggi, M. (2006). Inventories for male and female sexual dysfunctions. International Journal of Impotence Research, 18, $236-250$.

García - Portilla, M., Bascarán, P., Sáiz, M., Parellada, M., Bousoño, J., y Bobes, A. (2002). Banco de instrumentos básicos para la práctica de la psiquiatría clínica $\left(2^{\circ}\right.$ Ed.) Barcelona: Ars Médica.

Hernández, R., Fernández, C., y Baptista, P. (2006). Metodología de la investigación. México: MacGraw-Hill Interamericana.

Kloner, R. A. (2005). Erectile Disfunction and Cardiovascular Risk Factors. Urologic Clinics of North America, 32, 397-402.

Laumann, E. O., Paik, A., y Rosen, R. C. (1999). Sexual dysfunction in the United States: prevalence and predictors. Jama, 281, 537-544.

McVary, K. (2011). Contemporary treatment of erectile dysfunction: A Clinical Guide ( $1^{\circ}$ Ed.). Chicago: Humana Press.

Rosen, R., Riley, A., Wagner, G., Osterloh, I., Kirkpatrick, J., y Mishra, A. (1997). The international index of erectile function (IIEF): A multidimensional scale for assessment of erectile dysfunction. Urology, 49, 822-830. 


\section{Anexo}

Escala Internacional de Disfunción Eréctil adaptada a la población chilena IIEF-Ch

\section{Cuestionario Funcionamiento Sexual}

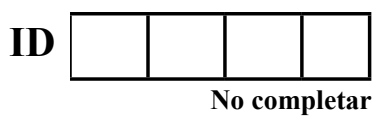

\section{Instrucciones:}

Encierre en un circulo según corresponda. Marque una sola opción por cada item

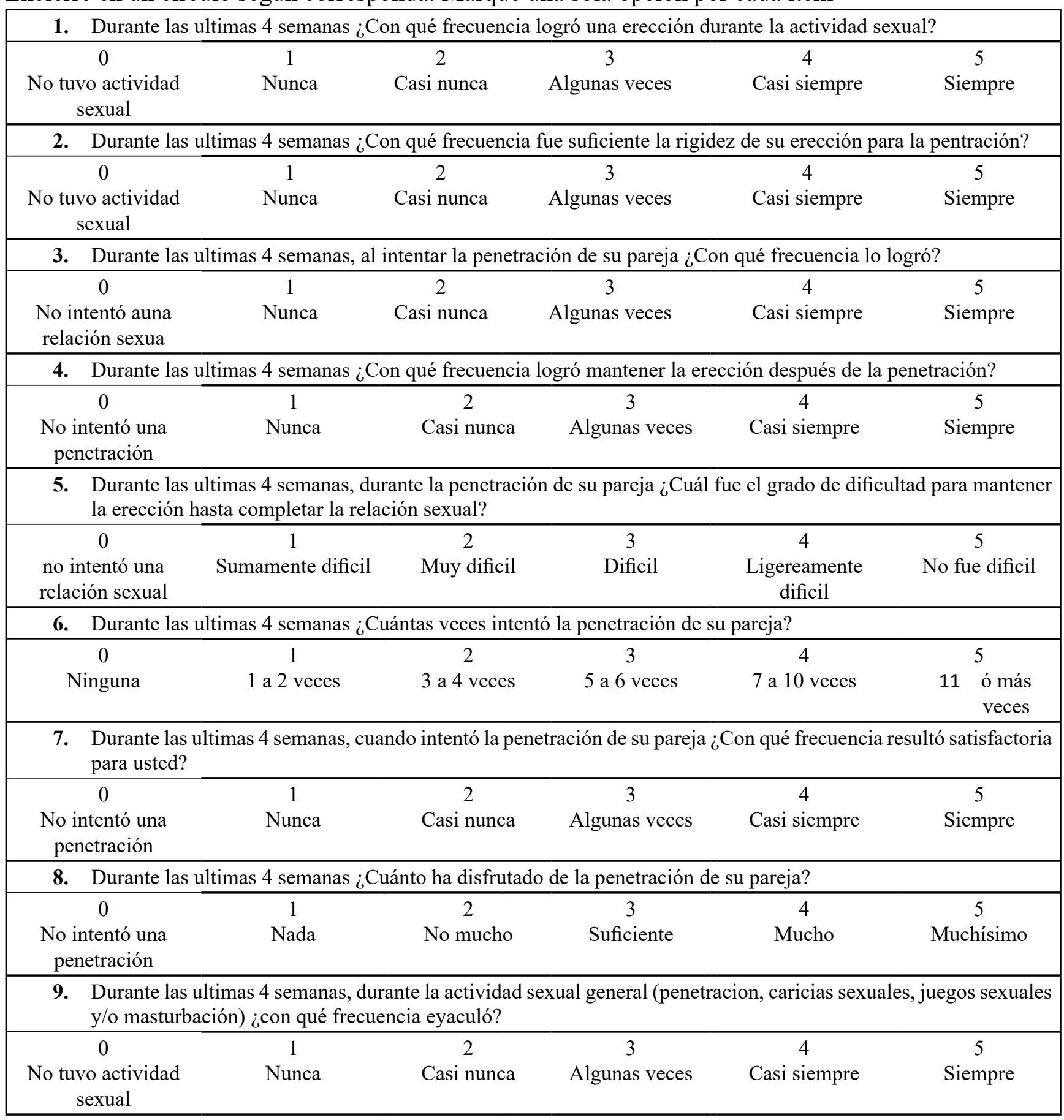


10. Durante las ultimas 4 semanas, durante la actividad sexual general (penetracion, caricias sexuales, juegos sexuales y/o masturbación) ¿Con qué frecuencia tuvo una sensación de orgasmo (con o sin eyaculación)?

\begin{tabular}{cccccc}
\hline 0 & 1 & 2 & 3 & 4 & 5 \\
$\begin{array}{c}\text { No tuvo actividad } \\
\text { sexual }\end{array}$ & Nunca & Casi nunca & Algunas veces & Casi siempre & Siempre \\
\hline
\end{tabular}

11. Durante las ultimas 4 semanas ¿Con qué frecuencia ha sentido el deseo de tener una experiencia sexual?

\begin{tabular}{|c|c|c|c|c|c|}
\hline & $\begin{array}{c}1 \\
\text { Nunca }\end{array}$ & $\begin{array}{c}2 \\
\text { Casi nunca }\end{array}$ & $\begin{array}{c}3 \\
\text { Algunas veces }\end{array}$ & $\begin{array}{c}4 \\
\text { Casi siempre }\end{array}$ & $\begin{array}{c}5 \\
\text { Siempre }\end{array}$ \\
\hline \multicolumn{6}{|c|}{ 12. Durante las ultimas 4 semanas ¿Cómo calificaria su nivel de deseo de tener una expriencia sexual? } \\
\hline 0 & 1 & 2 & 3 & 4 & 5 \\
\hline Ausente & Muy bajo & Bajo & Moderado & Elevado & Muy elevado \\
\hline \multicolumn{6}{|c|}{ 13. Durante las ultimas 4 semanas ¿Cuál ha sido el grado de satisfacción con su vida sexual en general? } \\
\hline & 1 & 2 & 3 & 4 & 5 \\
\hline & Muy satisfecho & $\begin{array}{l}\text { Moderadamente } \\
\text { satisfecho }\end{array}$ & $\begin{array}{c}\text { Más o menos } \\
\text { satisfecho }\end{array}$ & $\begin{array}{l}\text { Moderadamente } \\
\text { satisfecho }\end{array}$ & Muy satisfecho \\
\hline
\end{tabular}

14. Durante las ultimas 4 semanas ¿Cuál ha sido el grado de satisfacción experimentado por usted al penetrar a su pareja?

\begin{tabular}{|c|c|c|c|c|c|}
\hline 0 & 1 & 2 & 3 & 4 & 5 \\
\hline $\begin{array}{l}\text { No intentó una } \\
\text { pentración }\end{array}$ & Muy satisfecho & $\begin{array}{l}\text { Moderadamente } \\
\text { satisfecho }\end{array}$ & $\begin{array}{c}\text { Más o menos } \\
\text { satisfecho }\end{array}$ & $\begin{array}{c}\text { Moderadamente s } \\
\text { satisfecho }\end{array}$ & Muy satisfecho \\
\hline
\end{tabular}

15. Durante las ultimas 4 semanas ¿Cómo calificaría la confianza que tiene en poder lograr y mantener una erección?

\begin{tabular}{cccccc}
0 & 1 & 2 & 3 & 4 & 5 \\
Ausente & Muy bajo & Bajo & Moderado & Elevado & Muy elevado \\
\hline
\end{tabular}

